but this study does suggest that the implementation of CTOs is a cost-effective intervention and is economically advantageous to the local Trust.

Preconception interventions and resources for women with serious mental illness: a rapid evidence review

Katie Atmore*, Louise Howard and Abigail Easter

IoPPN King's College London

${ }^{\star}$ Corresponding author.

doi: 10.1192/bjo.2021.626

Aims. There is little research into evidence-based preconception interventions for women with serious mental illness (SMI). Women with SMI will have specific needs around preconception due to the complexities of the teratogenicity of medications, risk of mental illness relapse and higher levels of stigma around motherhood. If effectively delivered preconception care could mitigate these difficulties and improve outcomes for mother and baby. The aim of this research was therefore to determine to identify and describe studies evaluating preconception interventions for women of child-bearing age who have an existing SMI through searches of the peer-reviewed literature.

Method. A rapid review was conducted to search MEDLINE and PsychINFO databases from the year 2000 onwards for peerreviewed articles describing preconception interventions/ resources delivered prior to a pregnancy to women of childbearing age with a pre-existing existing serious mental illness (including schizophrenia, bipolar and eating disorders).

Result. A total of 592 results were returned from the searches and 576 of these remained after the removal of duplicates. 11 studies were included in the final narrative synthesis describing the following intervention types: Health warning (1), Health screening (1), Teratogen phone service (2), Psychiatric consultation (5), Family planning information (1) and Peripartum management plan (1). Interventions were delivered in Australia, UK, Italy, Germany, Netherlands, USA and Nigeria.

Conclusion. Though the included studies indicated that some efforts have been made globally to meet the preconception needs of women with SMI the numbers included in the studies tended to be low and reflective of small-scale service provision. Future studies utilising a randomised controlled trial design would lower the risk of bias and provide more generalisable evidence of effectiveness for these interventions. The results of this review were used to inform the development of a number of resources to aid the planning of healthy pregnancies in both women with SMI and the health professionals working with them.

\section{Relationship between bullying victimisation and post-traumatic stress disorder among public junior secondary school students in Abeokuta, Nigeria}

Sewanu Awhangansi ${ }^{1 *}$, Titilayo Salisu ${ }^{2}$, Oluwayemisi Akanji ${ }^{3}$, Adeniran Okewole ${ }^{4}$, Oladipo Sowunmi ${ }^{4}$, Sunday Amosu ${ }^{4}$, Increase Adeosun ${ }^{5}$ and Olugbenga Owoeye ${ }^{6}$

${ }^{1}$ Leicestershire partnership NHS Trust; ${ }^{2}$ Federal University of Agriculture (FUNAAB); ${ }^{3}$ Bowen University Teaching Hospital; ${ }^{4}$ Neuropsychiatric Hospital Aro; ${ }^{5}$ Babcock University Medical School and ${ }^{6}$ Federal Neuropsychiatric Hospital

${ }^{\star}$ Corresponding author.

doi: 10.1192/bjo.2021.627
Aims. To determine the relationship between bullying victimization and PTSD among students attending public Junior Secondary Schools in Abeokuta. The Prevalence of Bullying victimization and PTSD as well as some socio-demographic correlates were also assessed.

Method. About 411 junior students from five randomly selected public secondary schools were approached for the study and given consent forms to take home to their parents/guardians. Those who subsequently returned signed consent forms and who gave assent to participate in the study were administered the Socio-demographic questionnaire and the Multidimensional Peer Victimisation Scale (MDPVS). They were thereafter interviewed with the PTSD module of the MINI KID.

Result. A total of 351 students completed the study to yield a response rate of $85.4 \%$. The age range of the respondents was 9-17 years with mean (SD) of 12.48 (1.50) years. The gender distribution was $49.3 \%$ males and $50.7 \%$ females. $68.7 \%$ of the respondents were from a monogamous home, 22.2\% had divorced parents, $74.3 \%$ lived with both parents, and $6 \%$ reported being an only child. $14.8 \%$ of the respondents reported having experienced higher levels (moderate \& high) of victimization by peers. The mean score of the overall bullying victimization level was $9.6( \pm 6.5)$. Verbal victimization subscale had the highest mean score of $3.2( \pm 2.0)$, while physical victimization had the lowest mean of $1.9( \pm 2.1)$. Seventy $(19.9 \%)$ students admitted to the experience of a significant traumatic event, with only $7.1 \%$ of these meeting the current diagnosis of PTSD in the past month. There was no statistically significant association between bullying victimization and PTSD $(\chi 2=2.666 ; \mathrm{df}=2$; $\mathrm{p}=0.261$ ). Traumatic event experience was however significantly associated with high levels of bullying victimization experience $(\chi 2=4.266 ; \mathrm{p}=0.039)$. None of the assessed socio-demographic, familial or self-perceptual factors was found to be significantly associated with either bullying victimization or PTSD.

Conclusion. The experience of bullying victimization among secondary school students remains a prevailing problem in our local setting, as it is across the globe. Verbal bullying is the most common while physical bullying is the least common peer victimization experience in this study. The study points out that PTSD among high school students in our environment may be more prevalent than had previously been reported. Given the high rates of peer victimization experiences reported by students, there is a need for policy changes to make the school environment safer for students, thereby promoting their mental health.

The relationships between Big Five Personality dimensions, harmful psychoactive substance use and academic motivation among undergraduates in Nigeria

Busola Awolaran ${ }^{1 \star}$, Emmanuel Babalola ${ }^{2}$ and Peter Onifade ${ }^{3}$

${ }^{1}$ Kent and Medway NHS and Social Care Partnership Trust;

${ }^{2}$ Hywel Dda University Health Board and ${ }^{3}$ Drug Addiction

Treatment and Education and Research, Neuropsychiatric

Hospital Aro Abeokuta

${ }^{\star}$ Corresponding author.

doi: 10.1192/bjo.2021.628

Aims. The aim of this study was to determine the relationships between personality traits, stress perception, academic motivation and harmful use (use related harmful consequences) of alcohol, tobacco and cannabis among undergraduates in Southwestern Nigeria. 
Method. The study is a descriptive cross-sectional study conducted among students of randomly selected tertiary institutions in south western Nigeria. Ethical approval was obtained from the Research and Ethics Commitee of the Federal Neuropsychiatric Hospital Abeokuta Ogun State Nigeria. Permission to carry out the study was sought from the University authorities. A multistage cluster sampling selection of 850 respondents was done. Consenting students were administered socio-demographic questionnaire, WHO student's drug use questionnaire, the Big Five Personality Inventory (BFI-44), perceived stress scale-10 and academic motivation inventory.

Result. Seven hundred and eighty one completed questionnaires were analysed yielding a response rate of $92 \%$. There were $51 \%$ males and $49 \%$ females with a mean age of 23.3 years $(\mathrm{SD}=$ \pm 2.29 ), from monogamous family setting 591(75\%) and high socio-economic class $(65.8 \%)$. Of the respondents, $24.8 \%$ reported experience of use related harmful consequences such as engaging in quarrel or argument, unprotected sex and sex regretted the next day. There were significant associations between male gender $(\mathrm{p}=<0.001)$, urban residence $(\mathrm{p}=0.028)$, polygamous family setting $(p=0.002)$, high socioeconomic status $(p=0.026)$ and use related harmful consequences.

Multiple logistic regression showed that the odds of experiencing harmful consequences was less than 1 for agreeableness $(\mathrm{OR}=0.515, \mathrm{df}=1, \mathrm{p}=<0.001)$ and openness $(\mathrm{OR}=0.634, \mathrm{df}=1$, $\mathrm{p}=<0.028)$ but greater than 1 for extraversion $(\mathrm{OR}=1.525$, $\mathrm{df}=1, \mathrm{p}=<0.036$ ) personality dimensions. This implies that for a unit increase in agreeableness and openness scores, there were decreased odds $(8.6 \%$ and $79 \%$ respectively) of experiencing harmful consequences while there was increased odd (86\%) of experiencing harmful consequences from a unit increase in extraversion score.

Both binary and multiple regression analysis revealed that the odds of experiencing harmful consequences is greater than 1 for perceived stress score $(\mathrm{OR}=1.079, \mathrm{p}=<0.001)$ and less than 1 for academic motivation $(\mathrm{OR}=0.975, \mathrm{p}=<0.001)$. This means that perceived stress is positively associated with substance use and experience of harmful consequences while academic motivation is negatively associated with substance use and experience of harmful consequences

Conclusion. There were associations between certain sociodemographic factors, personality dimensions, stress perception and academic motivation with substance use and experience of harmful consequences. Thus, clinicians and researchers should consider these factors when designing preventive and treatment strategies.

\section{A narrative literature review of the typology of psychiatric emergency services in the UK \\ Dhruba Bagchi ${ }^{1 \star}$, George Tadros ${ }^{2}$ and Opeyemi Odejimi ${ }^{1}$ \\ ${ }^{1}$ Birmingham and Solihull Mental Health NHS Foundation Trust and ${ }^{2}$ Birmingham and Solihull Mental Health NHS Foundation Trust, Aston University \\ ${ }^{*}$ Corresponding author.}

doi: 10.1192/bjo.2021.629

Aims. This study aims to provide a detailed literature review of the different forms of Psychiatric Emergency Services currently available within the UK.

Background. 1 in 6 individuals have one form of mental health disorders. Mental health crisis resulting in an individual requiring access to Psychiatric Emergency Service (PES) can occur at any time. Psychiatric Emergency Service (PES) is described as one that provides an immediate response to an individual in crisis within the first 24 hours. Presently, several PESs are available in the UK with the aim of providing prompt and effective assessment, management and in some cases treatment and/or referral. Over the years, economic and political influences have greatly determined the service delivery models of PES. Indeed, these services vary in name, accessibility, structure, professionals involved, outcomes and many more.

Method. Electronic search of five key databases (MEDLINE, PsychINFO, EMBASE, AMED and PUBMED) was carried out to identify various models of PES in the UK. Various combinations of search terms were used and studies which met the inclusion criteria were selected. Studies were included if they were written in English, conducted within the United Kingdom, and described a form of PES. Search was not limited by years and this is to help have a comprehensive overview as well as show changes over time of the various models of psychiatric emergency services. Studies which did not meet any of the criteria detailed above were excluded.

Result. In total, 59 relevant studies were found which identified nine type of PES-Crisis resolution home treatment, police officer intervention, street triage, mental health liaison services in the Emergency Department, psychiatric assessment unit, integrated services, voluntary services and crisis house. There were more papers describing Crisis resolution home treatment services than the others. Furthermore, majority of the papers reported services within England than other countries within the UK.

Conclusion. All forms of PES are beneficial, particularly to mental health service users, but not without some shortcomings. There is a need to continue carrying out methodological research that evaluate impact, cost-effectiveness as well as identify methods of optimising the beneficial outcomes of all models of PES. This will inform researchers, educationist, policy makers and commissioners, service users and carers, service providers and many more on how to ensure current and future PES meet the needs as well as aid recovery of mental health service users.

\section{Mental wellbeing in doctors: the measure matters! development of a core outcome set for measuring wellbeing in doctors}

David Baldwin ${ }^{1 \star}$, Aimee O’Neill ${ }^{2}$, Julia Sinclair ${ }^{1}$ and Gemma Simons ${ }^{2}$

${ }^{1}$ Professor of Psychiatry and Head of Mental Health Group, University of Southampton Faculty of Medicine Honorary Professor of Psychiatry, University of Cape Town, South Africa Visiting Professor, Shandong Mental Health Centre, China and ${ }^{2}$ Centre for Workforce Wellbeing, University of southampton ${ }^{\star}$ Corresponding author.

doi: 10.1192/bjo.2021.630

Aims. To achieve a consensus Core Outcome Set for measuring mental wellbeing in doctors.

Hypothesis: A minimum set of valid, reliable and practical wellbeing measures is needed for doctors.

Background. The importance of doctors' mental wellbeing to everyone using Health Care is highlighted by the levels of burnout reported in doctors around the world. In 2019 a number of UK policy documents made recommendations for the wellbeing of doctors, but how those wellbeing interventions are evaluated needs to be defined. Core Outcome Sets are increasingly being used in medicine to prevent waste in research, by recommending the inclusion of a minimum set of valid, reliable and practical measures. An 